\title{
Kontrastmittel-Paravasate bei CT und MRT
}

Kontrastmittel-Paravasate stellen in der radiologischen Routine insbesondere bei CT-Untersuchungen ein nicht zu unterschätzendes Problem im klinischen Alltag dar. Bislang existieren jedoch keine einheitlichen radiologischen Empfehlungen oder Richtlinien, um bedrohliche ParavasatEreignisse frühzeitig zu erkennen und korrekt zu therapieren. Prof. Dr. Andreas G. Schreyer vom Universitätsklinikum Regensburg, Mit-Autor des aktuellen RöFoBeitrags „Kontrastmittel-Paravasat bei CT und MRT - Aktuelle Literaturübersicht und Behandlungsstrategien“, im Interview.
Herr Professor Schreyer, Sie haben sich jüngst mit Kontrastmittel-Paravasaten bei CT und MRT beschäftigt. Was hat Sie hierzu motiviert?

In der klinischen Routine, aber auch bei zahlreichen Fort- und Weiterbildungen ist mir aufgefallen, dass gerade zum Thema Paravasate viele unterschiedliche Meinungen und Empfehlungen zur weiteren Therapie kursieren. Als großer Freund der evidenzbasierten Medizin und von transparent erstellten Empfehlungen und Leitlinien habe ich daraufhin versucht, zusammen mit unseren plastischen Chirurgen, das Thema systematisch

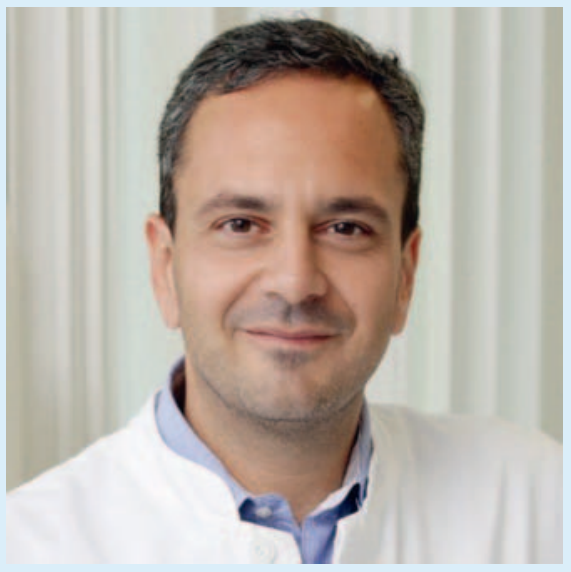

Prof. Dr. Andreas G. Schreyer 


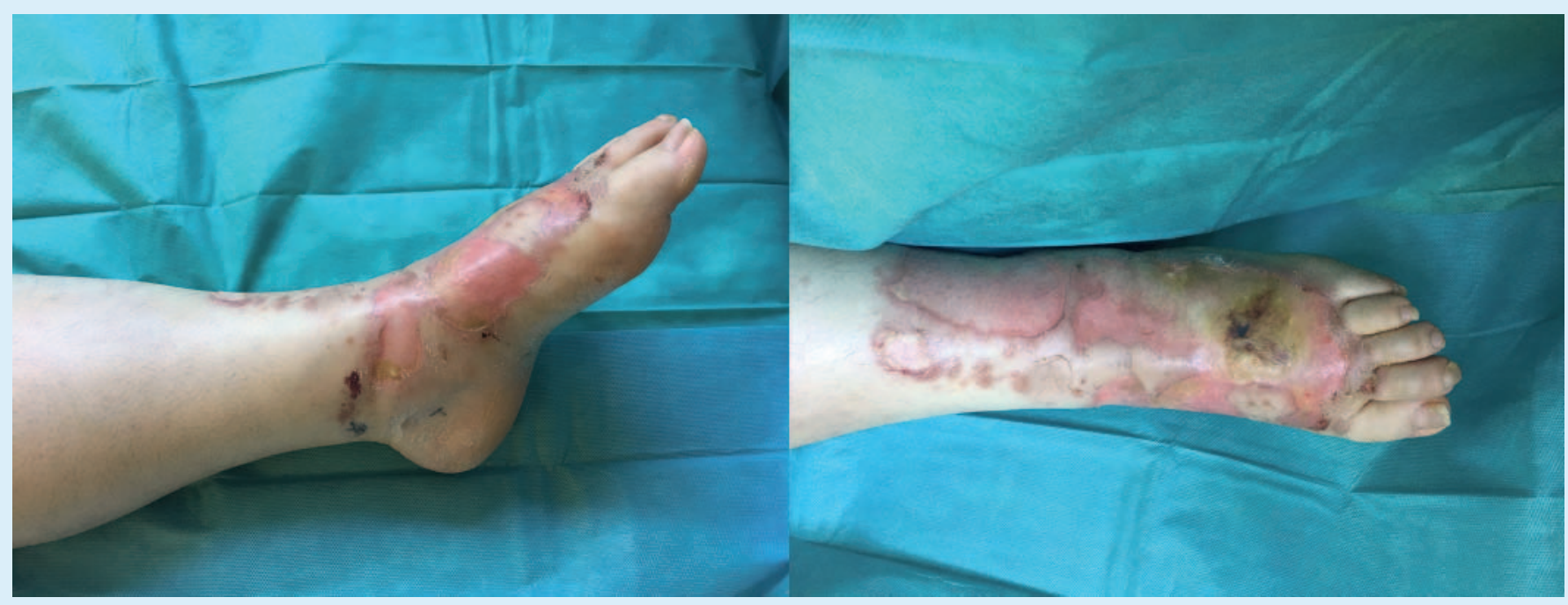

Nekrosen am Fußrücken eines adipösen Patienten nach Paravasat bei Kontrastmittelgabe bei einer Polytrauma-CT. Copyright: Prof. Dr. Andreas G. Schreyer.

aufzuarbeiten. Zu diesem Zweck haben wir systematisch die Literatur der letzten 35 Jahre durchgearbeitet und in den Kontext einer modernen chirurgischen Therapie bei Paravasaten gesetzt. Für uns kamen dabei erstaunliche Ergebnisse zu Tage: so haben wir unter anderem erkannt, dass die unklaren Therapieempfehlungen vor allem darauf zurückzuführen sind, dass die Literatur mehrheitlich auf alten Daten von vor 1990 fußt. Zudem wurden Empfehlungen für das Behandeln von Paravasaten bei z. B. Chemotherapeutika kritiklos auf Paravasate durch Röntgen-Kontrastmittel übertragen. Ich hoffe daher, dass wir mit unserem Manuskript und der systematisch aufgearbeiteten Literaturübersicht für mehr Klarheit sorgen können.

\section{Welche Paravasatkomplikationen gibt es und wie häufig treten diese auf?}

Das Spektrum der Komplikationen ist sehr breit - vom allenfalls milden Erythem bis hin zum Kompartmentsyndrom und Weichteilnekrosen. An dieser Stellte sollte berücksichtigt werden, dass moderne nicht-ionische Kontrastmittel eher selten schwerwiegende Komplikationen verursachen. Die Häufigkeit von Paravasaten wird in der Literatur zwischen 0,1 und $0,9 \%$ bei CT-Untersuchungen und lediglich $0,06 \%$ bei MRT-Untersuchungen angegeben. Diese Zahlen klingen zwar zunächst gering, da es sich v. a. bei der kontrastmittelgestützten $\mathrm{CT}$ jedoch um eine sehr häufige Untersuchung handelt, begegnet man Paravasaten und ihren Komplikationen in der Routine mehrmals wöchentlich.

\section{Können Sie bestimmte Therapieansätze bzw. Behandlungsstrategien empfehlen?}

Unsere systematische Literaturrecherche, dies deckt sich auch mit unserer klinischen Erfahrung, hat ergeben, dass bei Paravasatvolumina bis zu $150 \mathrm{ml}$ in der Regel kein weiteres chirurgisches Konsil nötig ist. Da bei den modernen Protokollen Kontrastmittelvolumina $>150 \mathrm{ml}$ kaum mehr verwendet werden, ist somit das Risiko für einen schweren, chirurgisch zu behandelnden Paravasatzwischenfall gering. Jedoch gilt dies nur eingeschränkt für Patienten mit vermindertem subkutanen Fettgewebe, wie wir es häufig bei kachektischen Patienten unter Chemotherapie sehen hier sollte man die weitere Überwachung intensivieren. So sollte beispielsweise bei abgeschwächtem peripheren Puls und Rekapillarisierung auch bei geringen Kontrastmittelvolumina ein chirurgisches Konsil sowie eine Fotodokumentation durchgeführt werden. Generell empfehlen wir bei Paravasaten konservative Maßnahmen wie Hochlagerung der Extremität über Herzhöhe, (Schienen)-Ruhigstellung und ggf. die Auflage von Coolpacks. Interessanterweise gibt es jedoch keine wirkliche wissenschaftliche Evidenz für diese konservativen Maßnahmen - es handelt sich hier eher um "good clinical practice“ und Erfahrungen aus der chirurgische Routine, die uns zu diesen Empfehlungen veranlassen. Basierend auf unserer Literaturrecherche sind invasive Maßnahmen, wie etwa Aspiration, Spülung oder manuelles Ausmelken bzw. die Injektion von Hyaluronsäure heute in der Regel nicht mehr indiziert - sie basieren auf veralteter Literatur, die sich vor allem auf Paravasate von Chemotherapeutika oder großen Mengen von Elektrolytlösungen bezieht. Für die klinische Praxis haben wir zusammen mit der Deutschen Gesellschaft der Plastischen, Rekonstruktiven und Ästhetischen Chirurgie ein Flowchart erstellt, das über die Webseite der Deutschen Röntgengesellschaft heruntergeladen werden kann.

\section{MEHR INFORMATIONEN}

Das Dokument zur Dokumentation von Paravasat-Ereignissen und das Flowchart können unter folgendem Link abgerufen werden: https://www. drg.de/de-DE/4556/kontrastmittelparavasate/ 